\title{
Yeasts from rumen of adult Zebu cows and fermentations of lignocellulosic materials
}

Eduardo Robson Duarte ( $\square$ duartevet@hotmail.com )

Universidade Federal de Minas Gerais https://orcid.org/0000-0002-2205-9412

Sabrina Miranda Lima

Instituto de Ciências Agrárias da Universidade Federal de Minas Gerais

Jualiane Costa Nere

Universidade Federal de Minas Gerais

Higor Alexandre Rodrigues Maia

Instituto Federal do Norte de Minas Gerais

Claudio Eduardo Silva Freitas

Universidade Federal de Minas Gerais

Aline Daniela Lopes Júlio

Universidade Federal de Minas Gerais

Flavia Oliveria Abrão

Instituto Federal Goiano - Campus Ceres

Vera Lúcia dos Santos

Universidade Federal de Minas Gerais

Júnio Cota Silva

Instituto de Ciências Agrárias da Universidade Federal de Minas Gerais

Research article

Keywords: Ruminal micobiota, Sugarcane bagasse, Submerged fermentation, Biomass production, Tropical pasture

Posted Date: May 20th, 2020

DOI: https://doi.org/10.21203/rs.2.22116/v2

License: (9) This work is licensed under a Creative Commons Attribution 4.0 International License. Read Full License 
The authors have withdrawn this preprint from Research Square 